\begin{tabular}{ccc}
\hline Jij & Journal of Mathematical Analysis and Modeling \\
jmam.sabapub.com & J Math Anal \& Model \\
ISSN 2709-5924 & (2020)1(1) : 64-76 \\
& & \\
\hline
\end{tabular}

\title{
Analysis of implicit type of a generalized fractional differential equations with nonlinear integral boundary conditions
}

\author{
SALEH S. RedhWAN ${ }^{a}$,* (i) ,SADIKALI L. ShaikH ${ }^{b}$ \\ ${ }^{a}$ Department of Mathematics, Dr. Babasaheb Ambedkar Marathwada University, \\ Aurangabad, India. \\ ${ }^{b}$ Department of Mathematics, Maulana Azad College of arts, Science and Commerce, \\ Aurangabad, India.
}

• Received: 05.10.2020 • Accepted/Published Online: 26.11.2020 • Final Version: 03.12.2020

\begin{abstract}
The given paper describes the implicit fractional differential equation with nonlinear integral boundary conditions in the frame of Caputo-Katugampola fractional derivative. We obtain an analogous integral equation of the given problem and prove the existence and uniqueness results of such a problem using the Banach and Krasnoselskii fixed point theorems. To show the effectiveness of the acquired results, convenient examples are presented.

Keywords: implicit fractional differential equation, fractional derivative and fractional integral, fixed point theorem.

2010 MSC: 35A22, 26A33, 34A08.
\end{abstract}

\section{Introduction}

The subject of fractional differential equations (FDEs) has lately developed as a motivating area of research. In reality, fractional derivatives kinds supply an excellent tool for the description of the memory and hereditary properties of different materials and operations. More researchers have established that FDEs show in many research scopes, such as physics, chemical technology, biotechnology, population dynamics, and economics. There has been considerable growth in FDEs involving many fractional derivatives such as Caputo, Riemann-Liouville, Hilfer, and Hadamard have been investigated and advanced by employing various tools from the nonlinear analysis. See the monographs of Podlubny [1], Kilbas et al. [2], Malinowska et al. [3], and some articles, for e.g., [4, 5, 6, 7, 8, 9, 10, 11, $12,13,14,15,16,17]$ and the references cited therein. The forward evolution of the FDEs have been caught much interest lately as study some results on the existence and uniqueness of solutions of various types of FDEs under different conditions have been studied by

\footnotetext{
*Corresponding author: saleh.redhwan909@gmail.com
}

(C) 2020 SABA. All Rights Reserved. 
many authors by employing fixed point techniques, see [18, 19, 20, 21, 22, 23, 24, 25, 26] and many other references.

In recent years [27] the researcher inserted a new fractional integral, which generalizes the Riemann-Liouville and Hadamard integrals into one form. For more properties of this new operator and similar operators, can be seen in $[28,29]$. The identical fractional derivatives were established in $[3,30,31]$ which named Katugampola fractional operators.

The existence and uniqueness results of FDEs involving Caputo-Katugampola derivative

$$
\begin{gathered}
\mathrm{CK}_{\mathfrak{D}_{0^{+}}^{\sigma ; \rho}} \omega(\vartheta)=\mathfrak{g}(\vartheta, \omega(\vartheta)), \quad \vartheta \in[0, \mathrm{~T}], \\
\omega^{(\mathrm{k})}(0)=\omega_{0}^{(\mathrm{k})}, \quad \mathrm{k}=0,1, \ldots, \mathrm{m}-1
\end{gathered}
$$

have been discussed using the Peano and Picard-Lindelö theorems in [32].

In [33], the author presented a new type of fractional operator as a generalization of the Caputo and Caputo-Hadamard fractional derivative operators. Also, he applied the Gronwall inequality type to obtain the uniqueness theorem of the problem (1.1)-(1.2).

In [34], the authors established the existence, uniqueness, and Ulam-Hyers stability results of boundary value problems (BVP) for an implicit FDEs with anti-periodic condition involving Caputo-Katugampola type

$$
\begin{gathered}
\mathrm{CK} \mathfrak{D}_{\mathrm{a}^{+}}^{\sigma ; \rho} \omega(\vartheta)=\mathfrak{g}\left(\vartheta, \omega(\vartheta), C K \mathfrak{D}_{a^{+}}^{\sigma ; \rho} \omega(\vartheta)\right), \vartheta \in \mathfrak{J}=[\mathrm{a}, \mathrm{T}], \\
\omega(\mathfrak{a})+\omega(\mathrm{T})=0,
\end{gathered}
$$

by applying some fixed point theorems and generalized Gronwall inequality.

Recently, there are some papers dealing with the qualitative properties of solutions of nonlinear FDEs by using techniques of nonlinear functional analysis see for e.g. [35, 36, $37,38,39,40,41]$.

Motivated by the previous results, we study in this paper a new type of BVP for implicit FDE that is

$$
\begin{gathered}
\mathrm{CK} \mathfrak{D}_{\mathrm{a}^{+}}^{\sigma ; \rho} \omega(\vartheta)=\mathfrak{g}\left(\vartheta, \omega(\vartheta), C K \mathfrak{D}_{a^{+}}^{\sigma ; \rho} \omega(\vartheta)\right), \vartheta \in \mathfrak{J}=[\mathrm{a}, \mathrm{T}], \\
\omega(\mathrm{a})=0, \omega(\mathrm{T})=\int_{a}^{\vartheta} \mathfrak{h}(\tau, \omega(\tau)) d \tau,
\end{gathered}
$$

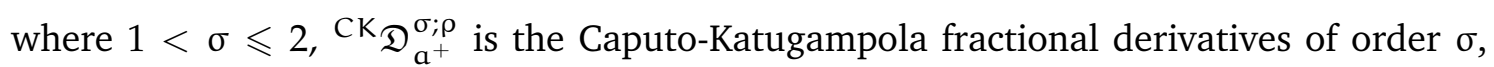
$\mathfrak{h}: \mathfrak{J} \times \mathbb{R} \longrightarrow \mathbb{R}$ and $\mathfrak{g}: \mathfrak{J} \times \mathbb{R} \times \mathbb{R} \longrightarrow \mathbb{R}$ are continuous functions.

The aim of this study is to extend and generalize some reported results in the literature through investigation in the existence and uniqueness of solutions for the given problem (1.3)-(1.4), in which the studied problem with nonlinear integral boundary condition is more general. Our analysis is depends on Banach's and Krasnoselskii's fixed point theorems [42].

The remainder of the paper is displayed as follows: In Section 2, we recall some essential definitions and properties which will be useful throughout this article and we proving some axiom lemmas which play a key role in the sequel. Section 3 contains certain sufficient conditions to corroborate the existence and uniqueness results of the 
problem (1.3)-(1.4) via fixed point techniques of Banach and Krasnoselskii. At the end, some examples are involved to illustrate the applicability of the obtained results in Section 4.

\section{Preliminaries}

For the sake of convenience of the readers, we present some background materials from fractional calculus theory and nonlinear analysis [27, 30, 33, 34, 42, 43] to facilitate the analysis of our problem (1.3)-(1.4).

Definition 2.1. [27] The Katugampola fractional integral of order $\sigma>0$ with $\rho>0$ is defined by

$$
\Im_{a^{+}}^{\sigma ; \rho} z(\vartheta)=\frac{\rho^{1-\sigma}}{\Gamma(\sigma)} \int_{a}^{\vartheta} \tau^{\rho-1}\left(\vartheta^{\rho}-\tau^{\rho}\right)^{\sigma-1} z(\tau) d \tau, \vartheta>a,
$$

if the integral exists, where, $\Gamma(\cdot)$ is a gamma function.

Definition 2.2. [30] The Katugampola fractional derivative of order $\sigma(n-1<\sigma<n)$ with $\rho>0$ is defined as

$$
\begin{aligned}
\mathfrak{D}_{a^{+}}^{\sigma ; \rho} z(\vartheta) & =\left(\vartheta^{1-\rho} \frac{d}{d \vartheta}\right)^{n} \mathfrak{I}_{a^{+}}^{n-\sigma ; \rho} z(\vartheta) \\
& =\frac{\gamma^{n} \rho^{\sigma-n+1}}{\Gamma(n-\sigma)} \int_{a}^{\vartheta} \tau^{\rho-1}\left(\vartheta^{\rho}-\tau^{\rho}\right)^{n-\sigma-1} z(\tau) d \tau, \vartheta>a,
\end{aligned}
$$

where $\gamma=\left(\vartheta^{1-\rho} \frac{\mathrm{d}}{\mathrm{d} \vartheta}\right)$ and $n=[\sigma]+1$.

Definition 2.3. [30] The Caputo-Katugampola fractional derivative of order $\sigma>0$ with $\rho$ $>0$ is defined by

$$
\mathrm{CK}_{\mathfrak{D}_{\mathrm{a}^{+}}^{\sigma ; \rho}}^{\sigma(\vartheta)}=\mathfrak{D}_{\mathrm{a}^{+}}^{\sigma ; \rho}\left[z(\vartheta)-\sum_{\mathrm{k}=0}^{\mathrm{n}-1} \frac{z_{\rho}^{(\mathrm{k})}(\mathrm{a})}{\mathrm{k} !}(\vartheta-\mathrm{a})^{\mathrm{k}}\right],
$$

where $z_{\rho}^{(k)}(\vartheta)=\left(\vartheta^{1-\rho} \frac{d}{d \vartheta}\right)^{k} z(\vartheta)$. In case $1<\sigma \leqslant 2$, and $z \in C^{2}(\mathfrak{J}, \mathbb{R})$, we have

$$
\mathrm{CK} \mathfrak{D}_{\mathrm{a}^{+}}^{\sigma ; \rho} z(\vartheta)=\mathfrak{D}_{\mathrm{a}^{+}}^{\sigma ; \rho}\left[z(\vartheta)-z(\mathrm{a})-z_{\mathfrak{p}}^{\prime}(\mathrm{a})(\vartheta-\mathrm{a})\right] .
$$

One has

$$
\mathrm{CK}_{\mathfrak{D}_{\mathrm{a}^{+}}^{\sigma ; \rho}} z(\vartheta)=\mathfrak{I}_{\mathrm{a}^{+}}^{\mathrm{n}-\sigma ; \rho}\left(\vartheta^{1-\rho} \frac{\mathrm{d}}{\mathrm{d} \vartheta}\right)^{\mathrm{n}} z(\vartheta) .
$$

Lemma 2.4. $[27,33] \mathfrak{I}_{\mathrm{a}^{+}}^{\sigma ; \rho}, C K \mathfrak{D}_{\mathrm{a}^{+}}^{\sigma ; \rho}$ are bounded operators from $\mathrm{C}[\mathrm{a}, \mathrm{T}]$ into $\mathrm{C}[\mathrm{a}, \mathrm{T}]$.

Lemma 2.5. [33] Let $\sigma>0, \beta>0, z \in \mathrm{C}^{1}[\mathrm{a}, \mathrm{T}]$. Then we have

$$
\mathfrak{I}_{\mathbf{a}^{+}}^{\sigma ; \rho} \mathfrak{I}_{\mathbf{a}^{+}}^{\beta ; \rho} \mathcal{Z}(\vartheta)=\mathfrak{I}_{\mathbf{a}^{+}}^{\sigma+\beta ; \rho} z(\vartheta), \quad C K_{\mathfrak{D}_{\mathbf{a}^{+}}^{\sigma ; \rho} \rho} \rho \mathfrak{I}_{\mathbf{a}^{+}}^{\sigma} z(\vartheta)=z(\vartheta) .
$$


Lemma 2.6. [27, 33] Let $\sigma>0$ and $\delta>n-1$. Then we have

$$
\begin{aligned}
& \mathfrak{I}_{a^{+}}^{\sigma ; \rho}\left(\vartheta^{\rho}-a^{\rho}\right)^{\delta}=\frac{\rho^{-\sigma} \Gamma(\delta+1)}{\Gamma(\delta+\sigma+1)}\left(\vartheta^{\rho}-a^{\rho}\right)^{\sigma+\delta}, \\
& \mathrm{CK}_{\mathfrak{D}} \mathrm{a}^{+}\left(\vartheta^{\rho}-\mathrm{a}^{\rho}\right)^{\delta}=\frac{\rho^{\sigma} \Gamma(\delta+1)}{\Gamma(\delta-\sigma+1)}\left(\vartheta^{\rho}-\mathrm{a}^{\rho}\right)^{\delta-\sigma},
\end{aligned}
$$

and

$$
\mathrm{CK}_{\mathfrak{D}_{\mathrm{a}^{+}}^{\sigma ; \rho}}\left(\vartheta^{\rho}-\mathrm{a}^{\rho}\right)^{\mathrm{k}}=0, \quad \sigma \geqslant 0, \mathrm{k}=0,1, \ldots, \mathrm{n}-1 .
$$

Lemma 2.7. [32, 34] Let $\sigma, \rho>0$ and $z \in C(\mathfrak{J}, \mathbb{R}) \cap C^{1}(\mathfrak{J}, \mathbb{R})$. Then the Caputo-Katugampola type FDE

$$
\mathrm{CK}_{\mathfrak{D}_{\mathrm{a}^{+}}^{\sigma ; \rho}}^{\sigma(\vartheta)}=0
$$

has a solution

$$
z(\vartheta)=c_{0}+c_{1}\left(\frac{\vartheta^{\rho}-a^{\rho}}{\rho}\right)+c_{2}\left(\frac{\vartheta^{\rho}-a^{\rho}}{\rho}\right)^{2}+\ldots+c_{n-1}\left(\frac{\vartheta^{\rho}-a^{\rho}}{\rho}\right)^{n-1}
$$

where $c_{i} \in \mathbb{R}, \mathfrak{i}=0,1,2, \ldots, n-1$ and $\mathrm{n}=[\sigma]+1$.

Moreover, if ${ }^{\mathrm{CK}} \mathfrak{D}_{\mathrm{a}^{+}}^{\sigma ; \rho} z \in \mathrm{C}(\mathfrak{J}, \mathbb{R}) \cap \mathrm{C}^{1}(\mathfrak{J}, \mathbb{R})$. Then

$$
\begin{aligned}
\mathfrak{I}_{\mathrm{a}^{+}}^{\sigma ; \rho} \mathrm{CK} \mathfrak{D}_{\mathrm{a}^{+}}^{\sigma ; \rho} z(\vartheta)= & z(\vartheta)+\mathrm{c}_{0}+\mathrm{c}_{1}\left(\frac{\vartheta^{\rho}-\mathrm{a}^{\rho}}{\rho}\right)+\mathrm{c}_{2}\left(\frac{\vartheta^{\rho}-\mathrm{a}^{\rho}}{\rho}\right)^{2} \\
& +\ldots .+\mathrm{c}_{n-1}\left(\frac{\vartheta^{\rho}-\mathrm{a}^{\rho}}{\rho}\right)^{\mathrm{n}-1},
\end{aligned}
$$

where $c_{i} \in \mathbb{R}, i=0,1,2, \ldots, n-1$ and $n=[\sigma]+1$.

Theorem 2.8. [42] (Banach fixed point theorem) Let (X, d) be a nonempty complete metric space with $\mathrm{F}: \mathrm{X} \rightarrow \mathrm{X}$ is a contraction mapping. Then map $\mathrm{F}$ has a fixed point.

Theorem 2.9. [42] (Krasnoselskii's fixed point theorem) Let $X$ be a Banach space, let $\Omega$ be a bounded closed convex subset of $X$ and let $F_{1}, F_{2}: \Omega \rightarrow \Omega$ a be mapping such that $F_{1} x+F_{2} y \in$ $\Omega$ for every pair $x, y \in \Omega$. If $F_{1}$ is contraction and $F_{2}$ is completely continuous, then there exists $z \in \Omega$ such that $\mathrm{F}_{1} z+\mathrm{F}_{2} z=z$.

\section{Existence and uniqueness theorems for (1.3)-(1.4)}

In this section, we give the results on the existence, the uniqueness of solution for problem (1.3)-(1.4) depending on Theorems 2.8, 2.9. The next lemma plays a necessary role in analysis our results.

Lemma 3.1. Let $1<\sigma \leqslant 2, \rho>0$ and $\varpi, \mathfrak{h} \in \mathrm{C}(\mathfrak{J}, \mathbb{R})$. Then a function $\omega$ is a solution of the Caputo-Katugampola type FDE

$$
C K_{\mathfrak{D}_{a^{+}}}^{\sigma ; \rho} \omega(\vartheta)=\varpi(\vartheta), \quad \vartheta \in \mathfrak{J}
$$




$$
\omega(a)=0, \quad \omega(T)=\int_{a}^{\vartheta} \mathfrak{h}(\tau) d \tau,
$$

if $\omega(\vartheta)$ satisfies the following fractional integral equation

$$
\begin{aligned}
\omega(\vartheta)= & \left(\frac{\vartheta^{\rho}-a^{\rho}}{T \rho-a^{\rho}}\right)\left[\int_{a}^{T} \mathfrak{h}(\tau) d \tau-\frac{\rho^{1-\sigma}}{\Gamma(\sigma)} \int_{a}^{T} \tau^{\rho-1}\left(T^{\rho}-\tau^{\rho}\right)^{\sigma-1} \varpi(\tau) d \tau\right] \\
& +\frac{\rho^{1-\sigma}}{\Gamma(\sigma)} \int_{a}^{\vartheta} \tau^{\rho-1}\left(\vartheta^{\rho}-\tau^{\rho}\right)^{\sigma-1} \varpi(\tau) d \tau .
\end{aligned}
$$

Proof. Applying $\mathfrak{I}_{\mathbf{a}^{+}}^{\sigma ; \rho}$ on both sides of (3.1, and employing Lemma (2.7), we get

$$
\omega(\vartheta)=c_{0}+c_{1}\left(\frac{\vartheta^{\rho}-a^{\rho}}{\rho}\right)+\frac{\rho^{1-\sigma}}{\Gamma(\sigma)} \int_{a}^{\vartheta} \tau^{\rho-1}\left(\vartheta^{\rho}-\tau^{\rho}\right)^{\sigma-1} \varpi(\tau) d \tau,
$$

where $c_{0}, c_{1} \in \mathbb{R}$. Take the limit of the equations (3.4) as $\vartheta \rightarrow a, \vartheta \rightarrow T$ respectively, it follows from the integral boundary conditions (3.2) that

$$
\mathrm{c}_{0}=0,
$$

and

$$
c_{1}=\frac{\rho}{T^{\rho}-a^{\rho}}\left[\int_{a}^{T} \mathfrak{h}(\tau) d \tau-\frac{\rho^{1-\sigma}}{\Gamma(\sigma)} \int_{a}^{T} \tau^{\rho-1}\left(T^{\rho}-\tau^{\rho}\right)^{\sigma-1} \varpi(\tau) d \tau\right] .
$$

Substitute (3.5) and (3.6) into (3.4), we obtain

$$
\begin{aligned}
\omega(\vartheta)= & \left(\frac{\vartheta^{\rho}-a^{\rho}}{T^{\rho}-a^{\rho}}\right)\left[\int_{a}^{T} \mathfrak{h}(\tau) d \tau-\frac{\rho^{1-\sigma}}{\Gamma(\sigma)} \int_{a}^{T} \tau^{\rho-1}\left(T^{\rho}-\tau^{\rho}\right)^{\sigma-1} \varpi(\tau) d \tau\right] \\
& +\frac{\rho^{1-\sigma}}{\Gamma(\sigma)} \int_{a}^{\vartheta} \tau^{\rho-1}\left(\vartheta^{\rho}-\tau^{\rho}\right)^{\sigma-1} \varpi(\tau) d \tau .
\end{aligned}
$$

The converse follows by Lemmas 2.5 and 2.6. The proof is completed.

As result of Lemma 3.1, we have the following Lemma:

Lemma 3.2. Assume that $\mathfrak{g}: \mathfrak{J} \times \mathbb{R} \times \mathbb{R} \rightarrow \mathbb{R}$ and $\mathfrak{h}: \mathfrak{J} \times \mathbb{R} \rightarrow \mathbb{R}$ are continuous and $\omega$ be a function. The problem (1.3)-(1.4) has a solution $\omega(\vartheta)$ if and only if $\omega(\vartheta)$ is a fixed-point of the operator $\mathrm{F}: \mathrm{C}(\mathfrak{J}, \mathbb{R}) \rightarrow \mathrm{C}(\mathfrak{J}, \mathbb{R})$ defined by

$$
\begin{aligned}
F \omega(\vartheta)= & \left(\frac{\vartheta^{\rho}-a^{\rho}}{T^{\rho}-a^{\rho}}\right)\left[\int_{a}^{T} \mathfrak{h}(\tau, \omega(\tau)) d \tau-\frac{\rho^{1-\sigma}}{\Gamma(\sigma)} \int_{a}^{T} \tau^{\rho-1}\left(T^{\rho}-\tau^{\rho}\right)^{\sigma-1} \mathbb{G}_{\omega}(\tau) d \tau\right] \\
& +\frac{\rho^{1-\sigma}}{\Gamma(\sigma)} \int_{a}^{\vartheta} \tau^{\rho-1}\left(\vartheta^{\rho}-\tau^{\rho}\right)^{\sigma-1} \mathbb{G}_{\omega}(\tau) d \tau .
\end{aligned}
$$

and $G_{\omega}(\vartheta):[a, T] \rightarrow \mathbb{R}$ be a function satisfying the functional equation

$$
\mathbb{G}_{\omega}(\vartheta):=\mathfrak{g}\left(\vartheta, \omega(\vartheta),{ }^{C K} \mathfrak{D}_{a^{+}}^{\sigma ; \rho} \omega(\vartheta)\right)
$$

Clearly, $\mathbb{G}_{\omega} \in \mathrm{C}[\mathfrak{J}, \mathbb{R}]$. In addition, by Lemma 2.4, $\mathrm{F} \omega \in \mathrm{C}[\mathfrak{J}, \mathbb{R}]$. 
Now, we present results on the existence and uniqueness of solution for the problem (1.3)-(1.4) depending on Theorems 2.8, 2.9. Assume that the functions $\mathfrak{g}: \mathfrak{J} \times \mathbb{R} \times \mathbb{R} \rightarrow \mathbb{R}$ and $\mathfrak{h}: \mathfrak{J} \times \mathbb{R} \rightarrow \mathbb{R}$ are continuous and satisfy the following conditions:

$\left(\mathrm{H}_{1}\right)$ There exists a constant $0<\mathrm{L}_{\mathfrak{g}}<1$ such that:

$$
\left|\mathfrak{g}\left(\vartheta, x_{1}, x_{2}\right)-\mathfrak{g}\left(\vartheta, y_{1}, y_{2}\right)\right| \leqslant L_{\mathfrak{g}}\left[\left|x_{1}-y_{1}\right|+\left|x_{2}-y_{2}\right|\right], \quad \forall \vartheta \in \mathfrak{J}, x_{i}, y_{\mathfrak{i}} \in \mathbb{R},(i=1,2) .
$$

$\left(\mathbf{H}_{2}\right)$ There exists a constant $0<\mathrm{L}_{\mathfrak{h}}<1$ such that:

$$
|\mathfrak{h}(\vartheta, x)-\mathfrak{h}(\vartheta, y)| \leqslant L_{\mathfrak{h}}|x-y|, \quad \forall \vartheta \in \mathfrak{J}, x, y \in \mathbb{R} .
$$

$\left(\mathbf{H}_{3}\right)$ The following inequaility holds

$$
\Upsilon:=\mathrm{L}_{\mathfrak{h}}(\mathrm{T}-\mathrm{a})+\frac{2 \mathrm{~L}_{\mathfrak{g}} \rho^{-\sigma}}{1-\mathrm{L}_{\mathfrak{g}}} \frac{\left(\mathrm{T}^{\rho}-\mathrm{a}^{\rho}\right)^{\sigma}}{\Gamma(\sigma+1)}<1
$$

Theorem 3.3. Suppose that $\left(H_{1}\right),\left(H_{2}\right)$ and $\left(H_{3}\right)$ hold. Then the problem (1.3)-(1.4) has a unique solution on $\mathfrak{J}$.

Proof. First, we show that $\mathrm{F} \mathcal{B}_{\mathrm{r}} \subseteq \mathcal{B}_{\mathrm{r}}$, such that be $\mathrm{F}: \mathrm{C}(\mathfrak{J}, \mathbb{R}) \rightarrow \mathrm{C}(\mathfrak{J}, \mathbb{R})$ defined by (3.7) and

$$
\mathcal{B}_{\mathrm{r}}=\{\omega \in \mathrm{C}(\mathfrak{J}, \mathbb{R}),\|\omega\| \leqslant \mathrm{r}\},
$$

with select $r \geqslant \frac{\Theta}{1-\gamma}$, where $\Upsilon<1$ and

$$
\Theta:=\left(\mu_{\mathfrak{h}}(\mathrm{T}-\mathrm{a})+\frac{2 \mu_{\mathfrak{g}} \rho^{-\sigma}}{1-\mathrm{L}_{\mathfrak{g}}} \frac{\left(\mathrm{T}^{\rho}-\mathrm{a}^{\rho}\right)^{\sigma}}{\Gamma(\sigma+1)}\right) .
$$

Set $\sup _{\vartheta \in \mathfrak{J}}|\mathfrak{g}(\vartheta, 0,0)|:=\mu_{\mathfrak{g}}<\infty$, and $\sup _{\vartheta \in \mathfrak{J}}|\mathfrak{h}(\vartheta, 0)|:=\mu_{\mathfrak{h}}$. For $\vartheta \in \mathfrak{J}$, with our hypotheses we have

$$
\begin{aligned}
|F \omega(\vartheta)| \leqslant & \left\{\left(\frac{\vartheta^{\rho}-a^{\rho}}{T^{\rho}-a^{\rho}}\right)\left[\int_{a}^{T}|\mathfrak{h}(\tau, \omega(\tau))| d \tau+\frac{\rho^{1-\sigma}}{\Gamma(\sigma)} \int_{a}^{T} \tau^{\rho-1}\left(T^{\rho}-\tau^{\rho}\right)^{\sigma-1}\left|G_{\omega}(\tau)\right| d \tau\right]\right. \\
& \left.+\frac{\rho^{1-\sigma}}{\Gamma(\sigma)} \int_{a}^{\vartheta} \tau^{\rho-1}\left(\vartheta^{\rho}-\tau^{\rho}\right)^{\sigma-1}\left|G_{\omega}(\tau)\right| d \tau\right\} .
\end{aligned}
$$

From $\left(\mathrm{H}_{1}\right)$, we have

$$
\begin{aligned}
\left|\mathbb{G}_{\omega}(\tau)\right| & =\left|\mathfrak{g}\left(\tau, \omega(\tau),{ }^{C K} \mathfrak{D}_{a^{+}}^{\sigma ;} \omega(\tau)\right)\right| \\
& \leqslant\left|\mathfrak{g}\left(\tau, \omega(\tau),{ }^{C K} \mathfrak{D}_{a^{+}}^{\sigma ; \rho} \omega(\tau)\right)-\mathfrak{g}(\tau, 0,0)\right|+|\mathfrak{g}(\tau, 0,0)| \\
& \leqslant L_{\mathfrak{g}}|\omega(\tau)|+\mathrm{L}_{\mathfrak{g}}\left|{ }^{C K} \mathfrak{D}_{\mathfrak{a}^{+}}^{\sigma ; \rho} \omega(\tau)\right|+\mu_{\mathfrak{g}} \\
& =\mathrm{L}_{\mathfrak{g}}|\omega(\tau)|+\mathrm{L}_{\mathfrak{g}}\left|\mathrm{G}_{\omega}(\tau)\right|+\mu_{\mathfrak{g}} .
\end{aligned}
$$

Since $L_{\mathfrak{g}}<1$ and for any $\omega \in \mathcal{B}_{\mathrm{r}}$, we get

$$
\left|G_{\omega}(\tau)\right| \leqslant \frac{\left(L_{\mathfrak{g}} r+\mu_{\mathfrak{g}}\right)}{1-\mathrm{L}_{\mathfrak{g}}} .
$$


From $\left(\mathrm{H}_{2}\right)$, we obtain

$$
\begin{aligned}
|\mathfrak{h}(\tau, \omega(\tau))| & \leqslant|\mathfrak{h}(\tau, \omega(\tau))-\mathfrak{h}(\tau, 0)|+|\mathfrak{h}(\tau, 0)| \\
& \leqslant L_{\mathfrak{h}}|\omega(\tau)|+\mu_{\mathfrak{h}} .
\end{aligned}
$$

For $\omega \in \mathcal{B}_{\mathrm{r}}$,

$$
|\mathfrak{h}(\tau, \omega(\tau))| \leqslant \mathrm{L}_{\mathfrak{h}} r+\mu_{\mathfrak{h}} .
$$

Consequently,

$$
\begin{aligned}
& |F \omega(\vartheta)| \\
& \leqslant\left\{\left(\frac{\vartheta^{\rho}-a^{\rho}}{T^{\rho}-a^{\rho}}\right)\left[\left(L_{\mathfrak{h}} r+\mu_{\mathfrak{h}}\right) \int_{a}^{T} d \tau+\frac{\left(L_{\mathfrak{g}} r+\mu_{\mathfrak{g}}\right)}{1-L_{\mathfrak{g}}} \frac{\rho^{1-\sigma}}{\Gamma(\sigma)} \int_{a}^{T} \tau^{\rho-1}\left(T^{\rho}-\tau^{\rho}\right)^{\sigma-1} d \tau\right]\right. \\
& \left.+\frac{\left(\mathrm{L}_{\mathfrak{g}} \mathrm{r}+\mu_{\mathfrak{g}}\right)}{1-\mathrm{L}_{\mathfrak{g}}} \frac{\rho^{1-\sigma}}{\Gamma(\sigma)} \int_{\mathfrak{a}}^{\vartheta} \tau^{\rho-1}\left(\vartheta^{\rho}-\tau^{\rho}\right)^{\sigma-1} \mathrm{~d} \tau\right\} \\
& \leqslant\left[\left(L_{\mathfrak{h}} r+\mu_{\mathfrak{h}}\right)(T-\mathfrak{a})+\frac{\left(\mathrm{L}_{\mathfrak{g}} r+\mu_{\mathfrak{g}}\right) \rho^{-\sigma}}{1-\mathrm{L}_{\mathfrak{g}}} \frac{\left(T^{\rho}-\mathfrak{a}^{\rho}\right)^{\sigma}}{\Gamma(\sigma+1)}\right] \\
& +\frac{\left(\mathrm{L}_{\mathfrak{g}} r+\mu_{\mathfrak{g}}\right) \rho^{-\sigma}}{1-\mathrm{L}_{\mathfrak{g}}} \frac{\left(\mathrm{T}^{\rho}-\mathrm{a}^{\rho}\right)^{\sigma}}{\Gamma(\sigma+1)} \\
& =\left(\mathrm{L}_{\mathfrak{h}}(\mathrm{T}-\mathrm{a})+\frac{2 \mathrm{~L}_{\mathfrak{g}} \rho^{-\sigma}}{1-\mathrm{L}_{\mathfrak{g}}} \frac{\left(\mathrm{T}^{\rho}-\mathrm{a}^{\rho}\right)^{\sigma}}{\Gamma(\sigma+1)}\right) \mathrm{r} \\
& +\left(\mu_{\mathfrak{h}}(\mathrm{T}-\mathrm{a})+\frac{2 \mu_{\mathfrak{g}} \rho^{-\sigma}}{1-\mathrm{L}_{\mathfrak{g}}} \frac{\left(\mathrm{T}^{\rho}-\mathrm{a}^{\rho}\right)^{\sigma}}{\Gamma(\sigma+1)}\right) \\
& =\Upsilon_{r}+\Theta<r \text {, }
\end{aligned}
$$

which gives $\|F \omega\|<$ r, i.e. $F \omega \in \mathcal{B}_{r}$. Moreover, by (3.7), and lammas 2.5, 2.6, we deduce

$$
\mathrm{CK}_{\mathfrak{D}^{2}}^{\sigma ; \rho} \mathrm{F} \omega(\vartheta)=\mathrm{CK}^{+} \mathfrak{D}_{\mathbf{a}^{+}}^{\sigma ; \rho} \mathfrak{I}_{\mathbf{a}^{+}}^{\sigma ; \rho} \mathbb{G}_{\omega}(\vartheta)=\mathbb{G}_{\omega}(\vartheta) .
$$

Since $G_{\omega}(\cdot)$ is continuous on $\mathfrak{J}$, the operator ${ }^{C K} \mathfrak{D}_{a^{+}}^{\sigma ; \rho} F \boldsymbol{\omega}(\vartheta)$ is continuous on $\mathfrak{J}$, that is $\mathrm{F} \mathcal{B}_{\mathrm{r}} \subseteq \mathcal{B}_{\mathrm{r}}$.

Second, we apply the Theorem 2.8 to show that $F$ has a fixed point. In fact, it sufficient to prove that $F$ is contraction. Let $\omega_{1}, \omega_{2} \in \mathcal{C}(\mathfrak{J}, \mathbb{R})$ and $\vartheta \in \mathfrak{J}$. Then

$$
\begin{aligned}
& \left|F \omega_{1}(\vartheta)-F \omega_{2}(\vartheta)\right| \\
\leqslant & \left(\frac{\vartheta^{\rho}-a^{\rho}}{T^{\rho}-a^{\rho}}\right)\left\{\int_{a}^{T}\left|\mathfrak{h}\left(\tau, \omega_{1}(\tau)\right)-\mathfrak{h}\left(\tau, \omega_{2}(\tau)\right)\right| d \tau\right. \\
& \left.+\frac{\rho^{1-\sigma}}{\Gamma(\sigma)} \int_{a}^{T} \tau^{\rho-1}\left(T^{\rho}-\tau^{\rho}\right)^{\sigma-1}\left|G_{\omega_{1}}(\tau)-G_{\omega_{2}}(\tau)\right| d \tau\right\} \\
& +\frac{\rho^{1-\sigma}}{\Gamma(\sigma)} \int_{a}^{\vartheta} \tau^{\rho-1}\left(\vartheta^{\rho}-\tau^{\rho}\right)^{\sigma-1}\left|G_{\omega_{1}}(\tau)-G_{\omega_{2}}(\tau)\right| d \tau .
\end{aligned}
$$


By $\left(\mathrm{H}_{1}\right)$, we get

$$
\begin{aligned}
& \left|G_{\omega_{1}}(\tau)-G_{\omega_{2}}(\tau)\right| \\
= & \left|\mathfrak{g}\left(\tau, \omega_{1}(\tau),{ }^{C K} \mathfrak{D}_{a^{+}}^{\sigma ; \rho} \omega_{1}(\tau)\right)-\mathfrak{g}\left(\tau, \omega_{2}(\tau),{ }^{C K} \mathfrak{D}_{a^{+}}^{\sigma ; \rho} \omega_{2}(\tau)\right)\right| \\
\leqslant & L_{\mathfrak{g}}\left|\omega_{1}-\omega_{2}\right|+L_{\mathfrak{g}}\left|{ }^{C K} \mathfrak{D}_{a^{+}}^{\sigma ; \rho} \omega_{1}(\tau)-C \mathfrak{D}_{\mathfrak{D}^{\sigma}}^{\sigma ; \rho} \omega_{2}(\tau)\right| \\
= & L_{\mathfrak{g}}\left|\omega_{1}-\omega_{2}\right|+\mathrm{L}_{\mathfrak{g}}\left|G_{\omega_{1}}(\tau)-G_{\omega_{2}}(\tau)\right|,
\end{aligned}
$$

which yields

$$
\left|G_{\omega_{1}}(\tau)-G_{\omega_{2}}(\tau)\right| \leqslant \frac{L_{\mathfrak{g}}}{1-L_{\mathfrak{g}}}\left|\omega_{1}-\omega_{2}\right|,
$$

and using $\left(\mathrm{H}_{2}\right)$, we obtain

$$
\left|\mathfrak{h}\left(\tau, \omega_{1}(\tau)\right)-\mathfrak{h}\left(\tau, \omega_{2}(\tau)\right)\right| \leqslant L_{\mathfrak{h}}\left|\omega_{1}-\omega_{2}\right| .
$$

The relations (3.13), (3.14) and (3.12) give

$$
\begin{aligned}
& \left\|F \omega_{1}-F \omega_{2}\right\| \\
\leqslant & \left(\frac{\vartheta^{\rho}-a^{\rho}}{T^{\rho}+a^{\rho}}\right)\left\{L_{\mathfrak{h}} \int_{a}^{T} d \tau\right. \\
& \left.+\frac{L_{\mathfrak{g}}}{1-\mathrm{L}_{\mathfrak{g}}} \frac{\rho^{1-\sigma}}{\Gamma(\sigma)} \int_{a}^{T} \tau^{\rho-1}\left(T^{\rho}-\tau^{\rho}\right)^{\sigma-1} \mathrm{~d} \tau\right\}\left\|\omega_{1}-\omega_{2}\right\| \\
& +\frac{\mathrm{L}_{\mathfrak{g}}}{1-\mathrm{L}_{\mathfrak{g}}} \frac{\rho^{1-\sigma}}{\Gamma(\sigma)} \int_{a}^{\vartheta} \tau^{\rho-1}\left(\vartheta^{\rho}-\tau^{\rho}\right)^{\sigma-1} \mathrm{~d} \tau\left\|\omega_{1}-\omega_{2}\right\| \\
\leqslant & {\left[\mathrm{L}_{\mathfrak{h}}(T-a)+\frac{2 \mathrm{~L}_{\mathfrak{g}} \rho^{-\sigma}}{1-\mathrm{L}_{\mathfrak{g}}} \frac{\left(T^{\rho}-a^{\rho}\right)^{\sigma}}{\Gamma(\sigma+1)}\right]\left\|\omega_{1}-\omega_{2}\right\| } \\
= & \curlyvee\left\|\omega_{1}-\omega_{2}\right\| .
\end{aligned}
$$

Since $\Upsilon<1$, the operator $F$ is contraction. Consequently, Theorem 2.8 shows that the problem (1.3)-(1.4) has a unique solution.

Our second existence result for the problem (1.3)-(1.4) is based on the Theorem 2.9.

Theorem 3.4. Suppose that $\left(H_{1}\right),\left(H_{2}\right)$ and $\left(H_{3}\right)$ hold. Then there exist at least one solution of the problem (1.3)-(1.4) on $\mathfrak{J}$.

Proof. Consider the operator $\mathrm{F}$ defined by (3.7). Set the ball $\mathcal{B}_{\mathrm{r}_{0}}:=\{\omega \in \mathrm{C}(\mathfrak{J}, \mathbb{R}):\|\omega\| \leqslant$ $r_{0}$, with $r_{0} \geqslant \frac{\Theta}{1-\gamma}$, where $\Theta$ and $\Upsilon$ is defined as in Theorem 3.3. Moreover, we define the operators $F_{1}$ and $F_{2}$ on $\mathcal{B}_{r_{0}}$ by

$$
F_{1} \omega(\vartheta)=\left(\frac{\vartheta^{\rho}-a^{\rho}}{T^{\rho}-a^{\rho}}\right)\left[\int_{a}^{T} \mathfrak{h}(\tau, \omega(\tau)) d \tau-\frac{\rho^{1-\sigma}}{\Gamma(\sigma)} \int_{a}^{T} \tau^{\rho-1}\left(T^{\rho}-\tau^{\rho}\right)^{\sigma-1} G_{\omega}(\tau) d \tau\right]
$$

and

$$
F_{2} \omega(\vartheta)=\frac{\rho^{1-\sigma}}{\Gamma(\sigma)} \int_{a}^{\vartheta} \tau^{\rho-1}\left(\vartheta^{\rho}-\tau^{\rho}\right)^{\sigma-1} G_{\omega}(\tau) d \tau
$$


Clearly, for any $\omega \in \mathrm{C}(\mathfrak{J}, \mathbb{R})$,

$$
F \omega(\vartheta)=F_{1} \omega(\vartheta)+F_{2} \omega(\vartheta), \quad \vartheta \in \mathfrak{J} .
$$

The proof will be divided into several stages as follows:

Stage 1: $F_{1} \omega_{1}+F_{2} \omega_{2} \in \mathcal{B}_{r_{0}}$ for every $\omega_{1}, \omega_{2} \in \mathcal{B}_{r_{0}}$.

Applying the same arguments in (3.10) and (3.11), we obtain

$$
\begin{gathered}
\left|G_{\omega_{1}}(\tau)\right| \leqslant \frac{L_{\mathfrak{g}} r_{0}+\mu_{\mathfrak{g}}}{1-L_{\mathfrak{g}}} \text {, and }\left|G_{\omega_{2}}(\tau)\right| \leqslant \frac{L_{\mathfrak{g}} r_{0}+\mu_{\mathfrak{g}}}{1-L_{\mathfrak{g}}}, \\
\left|\mathfrak{h}\left(\tau, \omega_{1}(\tau)\right)\right| \leqslant L_{\mathfrak{h}} r_{0}+\mu_{\mathfrak{h}} \text {, and }\left|\mathfrak{h}\left(\tau, \omega_{2}(\tau)\right)\right| \leqslant L_{\mathfrak{h}} r_{0}+\mu_{\mathfrak{h}} .
\end{gathered}
$$

For each $\omega_{1}, \omega_{2} \in \mathcal{B}_{\mathrm{r}_{0}}$ and $\vartheta \in \mathfrak{J}$, we have

$$
\begin{aligned}
& \left|F_{1} \omega_{1}(\vartheta)+F_{2} \omega_{2}(\vartheta)\right| \\
\leqslant & \left|F_{1} \omega_{1}(\vartheta)\right|+\left|F_{2} \omega_{2}(\vartheta)\right| \\
& \left(\frac{\vartheta^{\rho}-a^{\rho}}{T^{\rho}-a^{\rho}}\right)\left[\int_{a}^{T}\left|\mathfrak{h}\left(\tau, \omega_{1}(\tau)\right)\right| d \tau+\frac{\rho^{1-\sigma}}{\Gamma(\sigma)} \int_{a}^{T} \tau^{\rho-1}\left(T^{\rho}-\tau^{\rho}\right)^{\sigma-1}\left|G_{\omega_{1}}(\tau)\right| d \tau\right] \\
& +\frac{\rho^{1-\sigma}}{\Gamma(\sigma)} \int_{a}^{\vartheta} \tau^{\rho-1}\left(\vartheta^{\rho}-\tau^{\rho}\right)^{\sigma-1}\left|G_{\omega_{2}}(\tau)\right| d \tau \\
\leqslant & {\left[\left(L_{\mathfrak{h}}(T-a)+\frac{L_{\mathfrak{g}} \rho^{-\sigma}}{1-L_{\mathfrak{g}}} \frac{\left(T^{\rho}-a^{\rho}\right)^{\sigma}}{\Gamma(\sigma+1)}\right)+\frac{L_{\mathfrak{g}} \rho^{-\sigma}}{1-\mathrm{L}_{\mathfrak{g}}} \frac{\left(T^{\rho}-a^{\rho}\right)^{\sigma}}{\Gamma(\sigma+1)}\right] r_{0} } \\
& +\left[\left(\mu_{\mathfrak{h}}(T-a)+\frac{\mu_{\mathfrak{g}} \rho^{-\sigma}}{1-\mathrm{L}_{\mathfrak{g}}} \frac{\left(T^{\rho}-a^{\rho}\right)^{\sigma}}{\Gamma(\sigma+1)}\right)+\frac{\mu_{\mathfrak{g}} \rho^{-\sigma}}{1-\mathrm{L}_{\mathfrak{g}}} \frac{\left(T^{\rho}-a^{\rho}\right)^{\sigma}}{\Gamma(\sigma+1)}\right] \\
= & \left(\mathrm{L}_{\mathfrak{h}}(T-a)+\frac{2 \mathrm{~L}_{\mathfrak{g}} \rho^{-\sigma}}{1-\mathrm{L}_{\mathfrak{g}}} \frac{\left(T^{\rho}-a^{\rho}\right)^{\sigma}}{\Gamma(\sigma+1)}\right) \mathrm{r}_{0} \\
& +\left(\mu_{\mathfrak{h}}(T-a)+2 \frac{\mu_{\mathfrak{g}} \rho^{-\sigma}}{1-\mathrm{L}_{\mathfrak{g}}} \frac{\left(T^{\rho}-\mathfrak{a}^{\rho}\right)^{\sigma}}{\Gamma(\sigma+1)}\right) \\
= & r_{r_{0}+\Theta<r_{0},}
\end{aligned}
$$

which implies

$$
\left\|F_{1} \omega_{1}+F_{2} \omega_{2}\right\| \leqslant r_{0} .
$$

This proves that $F_{1} \omega_{1}+F_{2} \omega_{2} \in \mathcal{B}_{r_{0}}$ for every $\omega_{1}, \omega_{2} \in \mathcal{B}_{r_{0}}$.

Stage $2 F_{1}$ is a contration mapping on $\mathcal{B}_{r_{0}}$.

Since $F$ is contraction mapping as in Theorem 3.3, then $F_{1}$ is a contraction map too.

Stage 3. Here, we shall prove that operator $F_{2}$ is completely continuous on $\mathcal{B}_{r_{0}}$.

Obviously, $F_{2}$ is continuous due to the continuity of $G_{\omega}(\cdot)$.

Next, it is not difficult to conclude that

$$
\left\|F_{2} \omega\right\| \leqslant \frac{L_{\mathfrak{g}} r_{0}+\mu_{\mathfrak{g}}}{1-L_{\mathfrak{g}}} \frac{\rho^{-\sigma}}{\Gamma(\sigma+1)}\left(T^{\rho}-a^{\rho}\right)^{\sigma}<r_{0}
$$

due to definitions of $\Upsilon$ and $r_{0}$. This verifies that $F_{2}$ is uniformly bounded on $\mathcal{B}_{r_{0}}$

Finally, we show that $F_{2}$ maps bounded sets into equicontinuous sets of $C(\mathfrak{J}, \mathbb{R})$. 
Let $\vartheta_{1}, \vartheta_{2} \in \mathfrak{J}$, with $\vartheta_{1}<\vartheta_{2}$ and for any $\omega \in \mathcal{B}_{\mathrm{r}_{0}}$. Then we have

$$
\begin{aligned}
& \left|F_{2} \omega\left(\vartheta_{2}\right)-F_{2} \omega\left(\vartheta_{1}\right)\right| \\
= & \left|\frac{\rho^{1-\sigma}}{\Gamma(\sigma)} \int_{a}^{\vartheta_{2}} \tau^{\rho-1}\left(\vartheta_{2}^{\rho}-\tau^{\rho}\right)^{\sigma-1} G_{\omega}(\tau) d \tau-\frac{\rho^{1-\sigma}}{\Gamma(\sigma)} \int_{a}^{\vartheta_{1}} \tau^{\rho-1}\left(\vartheta_{1}^{\rho}-\tau^{\rho}\right)^{\sigma-1} \mathbb{G}_{\omega}(\tau) d \tau\right| \\
\leqslant & \frac{\rho^{1-\sigma}}{\Gamma(\sigma)} \int_{a}^{\vartheta_{1}} \tau^{\rho-1}\left[\left(\vartheta_{2}^{\rho}-\tau^{\rho}\right)^{\sigma-1}-\left(\vartheta_{1}^{\rho}-\tau^{\rho}\right)^{\sigma-1}\right]\left|G_{\omega}(\tau)\right| d \tau \\
& +\frac{\rho^{1-\sigma}}{\Gamma(\sigma)} \int_{\vartheta_{1}}^{\vartheta_{2}} \tau^{\rho-1}\left(\vartheta_{2}^{\rho}-\tau^{\rho}\right)^{\sigma-1}\left|G_{\omega}(\tau)\right| d \tau \\
\leqslant & \frac{L_{\mathfrak{g}} r_{0}+\mu_{\mathfrak{g}}}{1-L_{\mathfrak{g}}} \frac{\rho^{-\sigma}}{\Gamma(\sigma+1)}\left(\vartheta_{2}-\vartheta_{1}\right)^{\sigma} .
\end{aligned}
$$

As $\vartheta_{1} \longrightarrow \vartheta_{2}$ the right-hand side of the above inequality is not dependent on $\omega$ and tends to zero. Therefore,

$$
\left|F_{2} \omega\left(\vartheta_{1}\right)-F_{2} \omega\left(\vartheta_{2}\right)\right| \rightarrow 0, \forall\left|\vartheta_{2}-\vartheta_{1}\right| \rightarrow 0, \omega \in \mathcal{B}_{r_{0}} .
$$

This proves that $F_{2}$ is equicontinuous on $\mathcal{B}_{r_{0}}$. An application of Arzela-Ascoli Theorem shows that $F_{2}$ is relatively compact on $\mathcal{B}_{r_{0}}$. Hence all the assumptions of the Theorem 2.9 are satisfied. Thus, we deduce that the problem (1.3)-(1.4) has at least one solution on $\mathfrak{J}$.

\section{An example}

Here, we stipulate an interpretative example to support the obtained results.

Example 4.1. Consider the following implict FDE

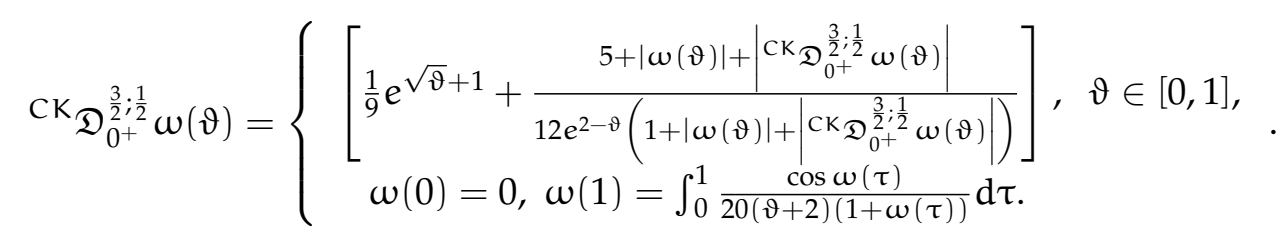

where $\sigma=\frac{3}{2}$ and $\rho=\frac{1}{2}$. Take

$$
\begin{gathered}
\mathfrak{g}(\vartheta, u, v)=\left[\frac{1}{9} e^{\sqrt{\vartheta}+1}+\frac{5+u+v}{12 e^{2-\vartheta}(1+u+v)}\right], \vartheta \in[0,1], u, v \in \mathbb{R}^{+}, \\
\mathfrak{h}(\vartheta, u)=\frac{\cos u}{20(\vartheta+2)(1+u)}, \vartheta \in[0,1], u \in \mathbb{R}^{+}
\end{gathered}
$$

Obviously, the functions $\mathfrak{g}, \mathfrak{h} \in \mathrm{C}([0,1], \mathbb{R})$. Moreover, $\sup _{\vartheta \in \mathfrak{J}}|\mathfrak{g}(\vartheta, 0,0)|=\frac{1}{9} e^{2}+\frac{5}{12 e}=$ $\mu_{\mathfrak{g}}<\infty$, and $\sup _{\vartheta \in \mathfrak{J}}|\mathfrak{h}(\vartheta, 0)|=\frac{1}{40}=\mu_{\mathfrak{h}}<\infty$. For each $u, v, u^{*}, v^{*} \in \mathbb{R}^{+}$and $\vartheta \in[0,1]$, we have

$$
\begin{aligned}
\left|\mathfrak{g}(\vartheta, \mathrm{u}, v)-\mathfrak{g}\left(\vartheta, \mathrm{u}^{*}, v^{*}\right)\right| & =\left|\frac{5+\mathrm{u}+v}{12 e^{2-\vartheta}(1+\mathrm{u}+v)}-\frac{5+\mathrm{u}^{*}+v^{*}}{12 e^{2-\vartheta}\left(1+\mathrm{u}^{*}+v^{*}\right)}\right| \\
& \leqslant \frac{1}{12 e^{2-\vartheta}}\left(\left|\mathrm{u}-\mathrm{u}^{*}\right|+\left|v-v^{*}\right|\right) \\
& \leqslant \frac{1}{12 e}\left(\left|\mathrm{u}-\mathrm{u}^{*}\right|+\left|v-v^{*}\right|\right)
\end{aligned}
$$


and

$$
\begin{aligned}
\left|\mathfrak{h}(\vartheta, \mathfrak{u})-\mathfrak{h}\left(\vartheta, \mathfrak{u}^{*}\right)\right| & =\left|\frac{\cos u}{20(\vartheta+2)(1+\mathfrak{u})}-\frac{\cos \mathfrak{u}^{*}}{20(\vartheta+2)\left(1+\mathfrak{u}^{*}\right)}\right| \\
& \leqslant \frac{1}{20}\left|\mathfrak{u}-\mathfrak{u}^{*}\right|
\end{aligned}
$$

Hence, the assumption $\left(\mathrm{H}_{1}\right)$ and $\left(\mathrm{H}_{2}\right)$ is satisfied with $\mathrm{L}_{\mathfrak{g}}=\frac{1}{12 e}, \mathrm{~L}_{\mathfrak{h}}=\frac{1}{20}$ respectively. We can easily check that $\left(\mathrm{H}_{3}\right)$. It can be seen by direct calculations that $\Upsilon=0.18458<1$. Since all the assumptions of Theorem (3.3) are obtained, therefore problem (4.1) has a unique solution.

\section{Conclusions}

In this article, we have studied a type of a nonlinear IFDE with the nonlinear integral boundary condition involving a Caputo-Katugampola fractional derivative. We have also established sufficient conditions ensuring existence, and uniqueness of solutions for a proposed proplem by applying some fixed point theorems. We confident the obtained results here will have a favorable impact on the evolution of more applications in applied sciences and engineering.

\section{Acknowledgment}

We thank the reviewers for their constructive remarks on our manuscript.

\section{References}

[1] Podlubny I (1999). "Fractional Differential Equations". Academic Press, San Diego.

[2] Kilbas AA , Srivastava HM and Trujillo JJ (2006). "Theory and applications of fractional differential equations". Elsevier Science Limited, 204.

[3] Malinowska AB, Odzijewicz T and Torres DFM (2015). "Advanced Methods in the Fractional Calculus of Variations". Springer, Berlin.

[4] Abdeljawad T (2011). On Riemann and Caputo fractional differences. Comput. Math. Appl. 62(3):16021611. https://doi.org/10.1016/j.camwa.2011.03.036

[5] Jarad F, Abdeljawad T and Baleanu D (2012). Caputo-type modification of the Hadamard fractional derivatives. Adv. Differ. Equ. 2012 (142): 8. https://doi.org/10.1186/1687-1847-2012-142

[6] Caputo M and Fabrizio M (2015). A new Definition of Fractional Derivative without Singular Kernel. Progr. Fract. Differ. Appl. 1(2): 73-85. https://doi.org/10.12785/pfda/010201

[7] Atangana A and Baleanu D (2016). New fractional derivatives with nonlocal and nonsingular kernel: theory and application to heat transfer model. Therm. Sci. 20(2): 763-69. https://doi.org/10.2298/TSCI160111018A

[8] Jarad F, Ug̃urlu E, Abdeljawad T and Baleanu D (2017). On a new class of fractional operators. Adv. Differ. Equ. 2017(1): 247. https://doi.org/10.1186/s13662-017-1306-z

[9] Jarad F, Abdeljawad T and Baleanu D (2017). On the generalized fractional derivatives and their Caputo modification. J. Nonlinear Sci. Appl. 10(5): 2607-2619.

[10] Abdeljawad T (2015). On conformable fractional calculus. J. Comput. Appl. Math. 279: 57-66. https://doi.org/10.1016/j.cam.2014.10.016

[11] Zhou Y, Jiao F and Li J (2009). Existence and uniqueness for fractional neutral differential equations with infinite delay. Nonlinear Anal. 71(7-8): 3249-3256. https:// doi.org/10.1016/j.na.2009.01.202

[12] Samko SG, Kilbas AA and Marichev OI (1993). "Fractional integrals and derivatives". Yverdon-les-Bains, Switzerland: Gordon and Breach Science Publishers, Yverdon,1. 
[13] Adjabi Y, Jarad F, Baleanu D and Abdeljawad T (2016). On Cauchy problems with Caputo Hadamard fractional derivatives. J. Comput. Anal. Appl. 21(4): 661-681.

[14] Khan A, Khan H, Gömez-Aguilar JF, and Abdeljawad T (2019). Existence and Hyers-Ulam stability for a nonlinear singular fractional differential equations with Mittag-Leffler kernel. Chaos Solitons Fractals 127: 422-427. https://doi.org/10.1016/j.chaos.2019.07.026

[15] Ali A, Shah K, Jarad F, Gupta V, Abdeljawad T (2019). Existence and stability analysis to a coupled system of implicit type impulsive boundary value problems of fractional-order differential equations. Adv. Differ. Equ. 2019(1): 101. https://doi.org/10.1186/s13662-019-2047-y

[16] Abdo MS, Abdeljawad T, Ali SM, Shah K and Jarad F (2020). Existence of positive solutions for weighted fractional order differential equations. Chaos Solitons Fractals 141 110341: 1-21. https://doi.org/10.1016/j.chaos.2020.110341

[17] Abdo MS, Abdeljawad T, Shah K and Jarad F (2020). Study of impulsive problems under Mittag-Leffler power law. Heliyon 6(10): e05109. https://doi.org/10.1016/j.heliyon.2020.e05109

[18] Abdo MS and Panchal SK (2018). Some new uniqueness results of solutions to nonlinear fractional integro-differential equations. Annals Pure Appl. Math. 16 (1): 345-352. https://doi.org/10.22457/apam.v16n2a11

[19] Abdo MS, Saeed AM, Wahash HA and Panchal SK (2019). On non-local problems for fractional integrodifferential equation in Banach space. European J. Scient. Res. 151(3): 320-334.

[20] Abdo MS and Panchal SK (2018). Fractional integro-differential equations involving $\psi$-Hilfer fractional derivative. Adv. Appl. Math. Mech. 11(2) :338-359. https://doi.org/10.4208/aamm.OA-2018-0143

[21] Abdo MS, Saeed AM and Panchal SK (2019). Caputo fractional integro-differential equation with nonlocal conditions in Banach space. Int. J. Appl. Math. 32(2): 279-288. https://dx.doi.org/10.12732/ijam.v32i2.9

[22] Anguraj A, Karthikeyan P, Rivero M and Trujillo JJ (2014). On new existence results for fractional integrodifferential equations with impulsive and integral conditions. Compu. Math. Appl. 66(12): 2587-2594. https://doi.org/10.1016/j.camwa.2013.01.034

[23] Jarad F, Abdeljawad $\mathrm{T}$ and Hammouch $\mathrm{Z}$ (2018). On a class of ordinary differential equations in the frame of Atangana-Baleanu fractional derivative. Chaos Solitons Fractals 117:16-20. https://doi.org/10.1016/j.chaos.2018.10.006

[24] Abdeljawad T (2017). Fractional operators with exponential kernels and a Lyapunov type inequality. Adv. Differ. Equ. 2017(1):313. https://doi.org/10.1186/s13662-017-1285-0

[25] Ameen R, Jarad F and Abdeljawad T (2018). Ulam stability for delay fractional differential equations with a generalized Caputo derivative. Filomat 32(15): 5265-5274. https://doi.org/10.2298/FIL1815265A

[26] Abdo MS, Shah K, Panchal SK and Wahash HA (2020). Existence and Ulam stability results of a coupled system for terminal value problems involving $\psi$-Hilfer fractional operator. Adv. Differ. Equ. 2020: 316. https://doi.org/10.1186/s13662-020-02775-x

[27] Katugampola UN (2011). New approach to a generalized fractional integral. Appl. Math. Comput. 218(3): 860-865.

[28] Herrmann R (2014). "Fractional Calculus: An Introduction for Physicists". World Scientific, River Edge, New Jerzey, 2 Eds.

[29] Jumarie G (2005). On the representation of fractional Brownian motion as an integral with respect to (dt) a. Appl. Math. Lett. 18(7): 739-748. https://doi.org/10.1016/j.aml.2004.05.014

[30] Katugampola UN (2014). A new approach to generalized fractional derivatives. Bull. Math. Anal. Appl. 6: $1-15$.

[31] Katugampola UN (2015). Mellin transforms of the generalized fractional integrals and derivatives. Appl. Math. Comput. 257: 566-580.

[32] Katugampola UN (2014). Existence and uniqueness results for a class of generalized fractional differential equations. Preprint. arXiv:1411.5229.

[33] Almeida R (2017). A Gronwall inequality for a general Caputo fractional operator. arXiv preprint arXiv:1705.10079.

[34] Redhwan SS, Shaikh SL and Abdo MS (2020). Implicit fractional differential equation with antiperiodic boundary condition involving Caputo-Katugampola type. AIMS MATHEMATICS 5(4): 3714-3730. https://DOI: 10.3934/math.2020240

[35] Abbas S, Benchohra M and Graef JR (2018). "Implicit Fractional Differential and Integral Equations: Existence and Stability". Walter de Gruyter: London, UK.

[36] Benchohra M, Bouriah S and Darwish MA (2017). Nonlinear boundary value problem for implicit dif- 
ferential equations of fractional order in Banach spaces. Fixed Point Theory 18 :457-470. https://DOI 10.24193/fpt-ro.2017.2.36

[37] Benchohra M, Bouriah S and Graef JR (2016). Nonlinear implicit differential equations of fractional order at resonance. Electron. J. Diff. Equ. 324: 1-10.

[38] Benchohra M and Lazreg JE (2013). Nonlinear fractional implicit differential equations. Commun. Appl. Anal. 17: 471-482.

[39] Benchohra M and Bouriah S (2015). Existence and stability results for nonlinear boundary value problem for implicit differential equations of fractional order. Moroccan J. Pure Appl. Anal. 1(1): 22-37. https://doi.org/ 10.7603/s40956-015-0002-9

[40] Alvarez E, Lizama C and Ponce R (2015). Weighted pseudo anti-periodic solutions for fractional integrodifferential equations in Banach spaces. Appl. Math. Comp. 259 :164-172.

[41] Ahmad B and Nieto JJ (2011). Anti-periodic fractional boundary value problems. Comput. Math. Appl. 62: 1150-1156. https://doi.org/10.1016/j.camwa.2011.02.034

[42] Burton TA and KirkÜ C (1998). A fixed point theorem of Krasnoselskii Schaefer type. Mathematische Nachrichten 189: 23-31.

[43] Sousa JVC and Oliveira EC 2017. A Gronwall inequality and the Cauchy-type problem by means of $\psi$-Hilfer operator. arXiv preprint arXiv:1709.03634. 